\title{
Evolución de los sistemas de crianza de cabras Criollas Cubanas en el contexto de la conservación del genotipo
}

\section{Rearing systems evolution of Cuban Creole goats in the genotype conservation context}

Manuel A. La O Arias,

Francisco Guevara Hernández ${ }^{\mathrm{b}}$,

Luis Alfredo Rodríguez Larramendic,

René Pinto Ruiz ${ }^{\mathrm{b}}$,

José Nahed Toral ${ }^{\mathrm{d}}$,

Alejandro Ley de Coss ${ }^{\mathrm{e}}$,

Luis Reyes Muro ${ }^{\mathrm{f} *}$

a Programa de Innovación Agropecuaria Local. Instituto de Investigaciones Agropecuarias "Jorge Dimitrov" (Cuba) y Red de Estudios para el Desarrollo Rural A.C. Villacorzo, Chiapas.

b Facultad de Ciencias Agronómicas. Campus V. Villaflores, Chiapas. Universidad Autónoma de Chiapas.

c Facultad de Ingeniería. Sede Villacorzo, Chiapas. Universidad de Ciencias y Artes de Chiapas.

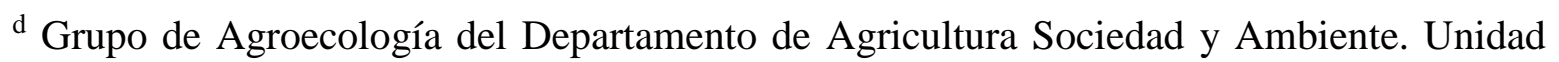
San Cristóbal de Las Casas, Chiapas. El Colegio de la Frontera Sur.

${ }^{\text {e }}$ Facultad de Ciencias Agrícolas, Campus IV. Huehuetán, Chiapas. Universidad Autónoma de Chiapas.

${ }^{\mathrm{f}}$ Campo Experimental Pabellón. Instituto Nacional de Investigaciones Forestales, Agrícolas y Pecuarias. Km 32.5 carr. Aguascalientes-Zacatecas. C. P. 20671. Tel. 01 (55) 38718700 ext 82513. 
*Autor de correspondencia: reyes.luis@inifap.gob.mx

\section{- Resumen:}

Se evaluó la evolución de los sistemas tradicionales de crianza de cabras criollas, durante el proceso de conservación de esta raza en la comunidad "26 de Julio", ubicada en la Subcuenca "Cautillo-Jiguaní" del Valle del Cauto, municipio de Jiguaní, provincia de Granma, Cuba. Se evaluaron 13 casos, con 18 variables, respecto a tamaño del hato, manejo, producción y eficiencia. Se definieron tres momentos de evaluación: a) inicio del proceso de conservación, estado de referencia - años 2002 y 2003 -, b) periodo de acompañamiento institucional, - años, 2005 y 2006 - y c) periodo de autogestión, -años 2008 y 2009 -. Se empleó el modelo estadístico multivariado de medición de impacto (MEMI). El impacto del proceso de conservación de la cabra criolla se resumió en tres dimensiones: a) tenencia y comercialización, b) eficiencia productiva y c) autoconsumo, las cuales explicaron el $82 \%$ de la varianza total. Según el comportamiento de los índices de impacto en estas tres dimensiones, las tipologías de sistemas estudiados mostraron tendencias diferentes en su evolución, con mayor capacidad de crecimiento en los sistemas de fincas, comparados con los sistemas de patios y parcelas. No obstante, las tipologías desarrollaron procesos de crecimiento, colapso y renovación, con capacidad para absorber creativamente los cambios y conservar sus racionalidades socioeconómicas.

- Palabras clave: Sistemas tradicionales, Cabras criollas, Conservación.

\section{- Abstract}

The evolution of the traditional rearing systems of Creole goats was assessed during the preservation process of this breed in the community "26 de Julio". This community is located in the sub-basin "Cautillo-Jiguaní" of Cauto Valley, Jiguaní municipality, Granma province, Cuba. For studying the systems, 13 cases and 18 variables related with size, management, production and efficiency were considered. Three assessment moments were defined: a) beginning of the preservation process, reference state - yr 2002 and 2003 -, b) period of institutional accompaniment, - yr, 2005 and 2006 - and c) self-management period, -yr 2008 and 2009 -. The multivariate statistical model for impact measuring $(\mathrm{MEMI})^{(1)}$ was used. The impact of the preservation process of Creole goat comprised three dimensions: tenure and commercialization, productive efficiency and self-subsistence, which explained the $82 \%$ of the total variance. According to the impact indexes in these three dimensions, the typology of studied systems showed difference tendencies on their evolution, with higher growth capacity by farm systems compared with those of backyards and plots. However, both typologies developed collapse and renewal growth processes showing capacity for absorbing the changes and preserve their socio-economical rationalities.

- Key words: Traditional systems, Creole goats, Preservation. 
Recibido el 10/03/2017

Aceptado el 11/08/2017

\section{N Introducción}

La cabra criolla ha formado parte del sistema de vida rural en Cuba, desde el comienzo de la colonización. La evidencia histórica demuestra que el propio Colón transportó los primeros ejemplares en sus viajes ${ }^{(2)}$. Sin embargo, la crianza caprina no ha ocupado un lugar relevante como actividad productiva dentro de la estructura económica agropecuaria histórica en Cuba, por tal motivo, esta raza se asocia a sistemas tradicionales de crianza.

Hasta la segunda mitad del siglo XX, los conceptos de desarrollo agropecuario en Cuba no estimularon iniciativas a favor de los genotipos criollos, en especial de las cabras. Las estrategias relacionadas con la especie caprina siguieron los principios hegemónicos de la Revolución Verde, y favorecieron la introducción de genotipos especializados, los que paulatinamente desplazaron a las cabras criollas. En el año 2003, el grupo de trabajo convocado por la Comisión Nacional de Recursos Genéticos de la República de Cuba, declaró oficialmente en peligro de extinción a la cabra criolla cubana ${ }^{(3)}$.

La $\mathrm{O}^{(4)}$, luego de explorar 48 presuntos rebaños criollos de la región del Valle del Cauto, informaron la existencia de la única población de cabras criollas de la zona en la comunidad "26 de Julio". Esta comunidad se convirtió en escenario del programa de conservación de la cabra criolla cubana, y la experiencia en caso relevante sobre la conservación de un genotipo criollo, avalado por instituciones internacionales, y con enfoques de investigación y acción participativas.

Este proceso de conservación es multifactorial. Implica una intervención externa que necesariamente impacta en la crianza. Esto es especialmente relevante por la profunda relación que existe entre la supervivencia de este genotipo y sus sistemas de crianza. En los sistemas tradicionales, interactúan componentes socioproductivos, con el objetivo de integrarse a estrategias de supervivencia campesina, conceptualización que no siempre es congruente con las estrategias de intervención multifactorial, por la complejidad metodológica que implica la concepción holística de la acción. 
Ante este desafío, se necesitan modelos longitudinales (que implican diferentes periodos), capaces de conectar las variaciones conjuntas de dichos componentes en el tiempo, o sea, la coevolución de sus variables. Un esfuerzo en este sentido es el Modelo Estadístico Multivariado de Medición de Impacto (MEMI) promovido por Torres et al ${ }^{(1)}$.

En el presente trabajo se realiza una exploración de las implicaciones para los sistemas tradicionales de crianza de las intervenciones institucionales durante el proceso de conservación de la cabra criolla, mediante un análisis longitudinal (antes, durante y después). Estos resultados son relevantes para la comprensión del cambio en los sistemas tradicionales, desde la perspectiva de la complejidad, y para el diseño de nuevas estrategias de acompañamiento institucional.

\section{Material y métodos}

\section{- Diseño general de la investigación •}

Se realiza una investigación exploratoria y longitudinal apoyada en modelos multivariados para el análisis de sistemas (Figura 1). Los periodos de estudio centran el acompañamiento institucional como hito para definir un antes, durante y después. El objeto de estudio fueron los sistemas tradicionales de crianza y su evolución en el contexto del acompañamiento institucional para la conservación de las cabras criollas. Se consideró la totalidad de los sistemas de crianza implicados en este proceso de conservación (13 sistemas) y la clasificación de los mismos en tipologías previamente establecidas ${ }^{(4)}$.

Las variables controladas dentro de los sistemas de crianza se levantaron a partir de registros establecidos por el programa de conservación y registros individuales de los criadores. En cada caso se controló su comportamiento en correspondencia con los periodos evaluados. 
Figura 1: Diseño general de la investigación

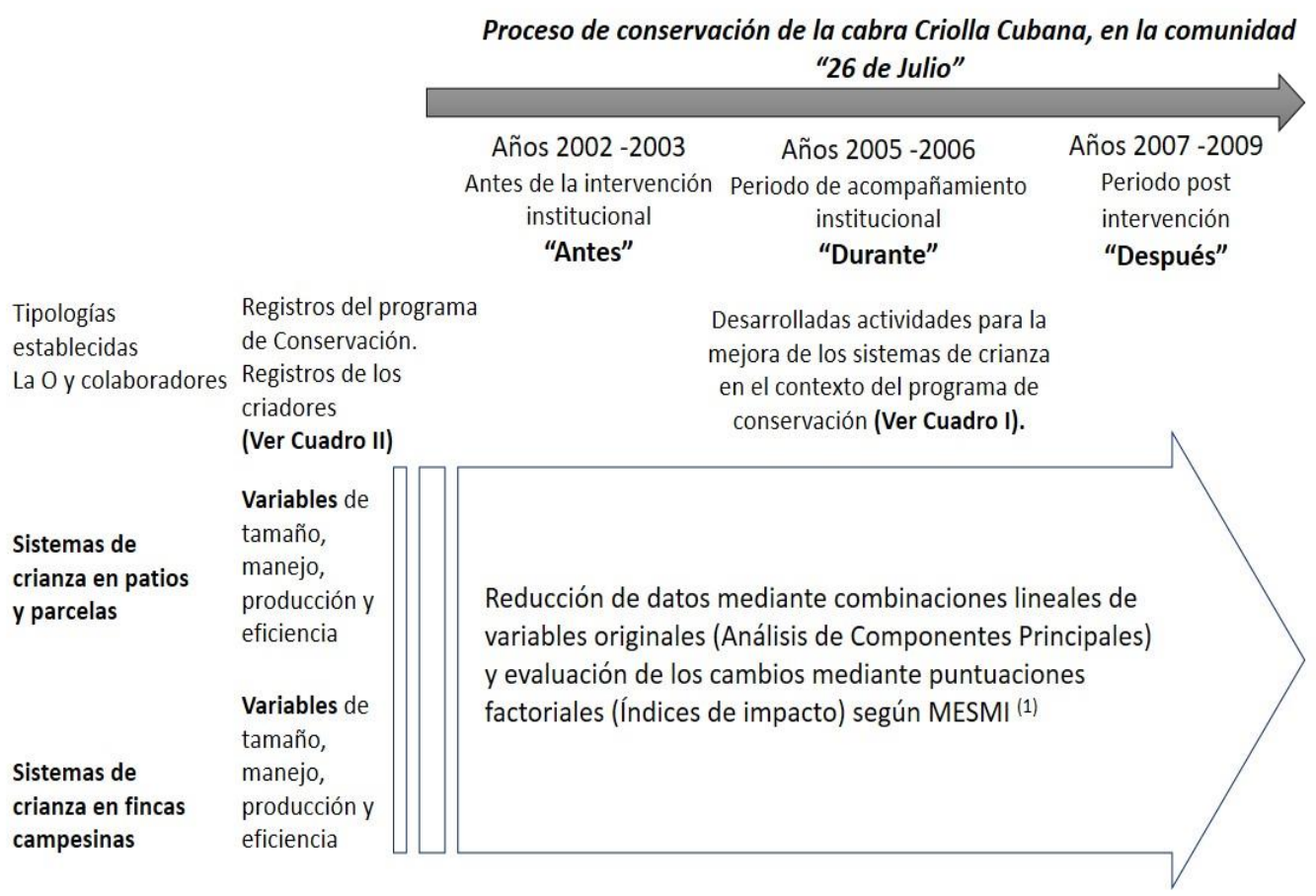

\section{- Área y casos de estudio}

La investigación se realizó en la comunidad "26 de Julio" en el contexto del proceso de conservación de la cabra criolla cubana. Esta comunidad se ubica en la Sub-cuenca “Cautillo-Jiguaní" del Valle del Cauto, municipio de Jiguaní, provincia de Granma, Cuba.

Para el estudio se seleccionaron 13 sistemas de crianza, que representan la totalidad de los implicados en todas las etapas del proceso de conservación. Los mismos respondieron a las dos tipologías de crianza definidas por $\mathrm{La} \mathrm{O}^{(4)}$. Las cuales se describen a continuación:

Sistemas de crianza familiar en patios y parcelas (7 casos). Se caracterizan por pequeños rebaños de hasta 13 reproductoras, con parcelas de 1.23 ha promedio; basan su crianza en la utilización de áreas marginales.

Sistemas de crianza familiar en fincas (6 casos). Se caracterizan por rebaños medianos de 34 reproductoras, el área total promedio de las unidades fue de 8.24 ha y utilizan 1.97 ha para pastoreo, con una menor dependencia de las áreas marginales para el pastoreo. 


\section{- Temporalidad para el análisis longitudinal}

Se definieron tres momentos o periodos de evaluación:

a) Inicio del proceso de conservación "Antes" (años 2002 y 2003). Considerado como periodo de referencia sin intervención institucional.

b) Periodo de acompañamiento institucional "Durante" (años, 2005 y 2006). Periodo incluido dentro del proceso de conservación de la cabra criolla en la comunidad, con actividades de investigación, acción participativa para le mejora de los sistemas de crianza y acompañamiento técnico, logístico y político de instituciones (Cuadro 1).

c) Periodo post acompañamiento "Después" (años 2008 y 2009). Retirada del apoyo institucional. Se realiza la investigación de seguimiento.

Cuadro 1: Actividades y cambios desarrollados en los sistemas de crianza durante el periodo de acompañamiento institucional

\begin{tabular}{|c|c|c|c|}
\hline \multirow[t]{2}{*}{ Adecuación } & \multirow{2}{*}{$\begin{array}{l}\text { Forma de acompañamiento } \\
\text { institucional } \\
\text { Método de desarrollo } \\
\end{array}$} & \multicolumn{2}{|c|}{$\begin{array}{l}\text { Asimilación } \\
\text { Tipología }\end{array}$} \\
\hline & & $\mathbf{I}$ & II \\
\hline \multicolumn{4}{|l|}{ Fortalecimiento de la base alimentaria } \\
\hline $\begin{array}{l}\text { Establecimiento de áreas forrajeras en los } \\
\text { sistemas de crianza. }\end{array}$ & $\begin{array}{l}\text { Selección participativa en feria de } \\
\text { diversidad de pastos. }\end{array}$ & - & ++ \\
\hline Fomento del algarrobo. & Rescate del conocimiento local. & ++ & ++ \\
\hline \multicolumn{4}{|l|}{ Mejoras al control de la reproducción } \\
\hline Establecimiento de campañas reproductivas. & & + & ++ \\
\hline $\begin{array}{l}\text { Utilización del efecto macho como práctica de } \\
\text { manejo de la reproducción. }\end{array}$ & $\begin{array}{l}\text { Experimentación participativa en } \\
\text { el rebaño núcleo. }\end{array}$ & - & + \\
\hline Sistema de monta dirigida. & & + & +++ \\
\hline \multicolumn{4}{|l|}{ Sistema de control parasitario } \\
\hline $\begin{array}{l}\text { Aplicación estratégica de antihelmínticos. } \\
\text { Selección negativa de máximos acumuladores } \\
\text { de parásitos. }\end{array}$ & $\begin{array}{l}\text { Experimentación participativa en } \\
\text { el rebaño núcleo. }\end{array}$ & + & $\begin{array}{l}++ \\
++\end{array}$ \\
\hline \multicolumn{4}{|c|}{ Adecuación del esquema de obtención y comercialización de la producción } \\
\hline $\begin{array}{l}\text { Sacrificio y comercialización de los machos } \\
\text { en crecimiento teniendo en cuenta la curva de } \\
\text { crecimiento. }\end{array}$ & $\begin{array}{l}\text { Experimentación participativa en } \\
\text { todos los rebaños. }\end{array}$ & ++ & +++ \\
\hline $\begin{array}{l}\text { Comercialización de reproductores registrados } \\
\text { (valor agregado). }\end{array}$ & $\begin{array}{l}\text { Experimentación participativa en } \\
\text { el rebaño núcleo. }\end{array}$ & + & +++ \\
\hline \multicolumn{4}{|c|}{ 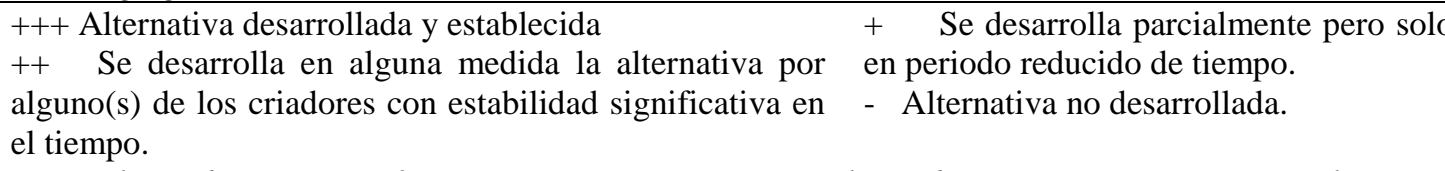 } \\
\hline II. Tipología de crianza en fincas & I. Tipología de crianzas en patic & $s$ y $p$ & \\
\hline
\end{tabular}

Fuente: La O $^{(4)}$ 


\section{- Variables consideradas para evaluar la evolución de los sistemas}

Para la evaluación del desarrollo de los sistemas de crianza se estudiaron variables relacionadas con su tamaño, manejo, producción y eficiencia (Cuadro 2). Estas fueron las variables originales incorporadas en las matrices de datos para el seguimiento. La interpretación de la evolución de los sistemas se realizó a partir de las componentes, resultantes de combinaciones lineales de ellas, establecidas por medio del modelo multivariado.

Cuadro 2: Variables utilizadas en los estudios de seguimiento de los sistemas familiares de crianza

\begin{tabular}{|c|c|c|c|}
\hline Tamaño & Manejo & Producción & Eficiencia \\
\hline $\begin{array}{c}\text { Cantidad de } \\
\text { reproductoras (cabezas) }\end{array}$ & $\begin{array}{l}\text { Área de compensación } \\
\text { o forrajera (ha) }\end{array}$ & $\begin{array}{c}\text { Consumo doméstico de } \\
\text { productos caprinos (kg } \\
\text { peso vivo). }\end{array}$ & $\begin{array}{l}\text { Producción/insumos } \\
\text { Relación entre el valor } \\
\text { de la producción y el } \\
\text { valor de los insumos }\end{array}$ \\
\hline $\begin{array}{c}\text { Cantidad de sementales } \\
\text { (cabezas) }\end{array}$ & $\begin{array}{l}\text { Dosis antiparasitarias } \\
\text { (dosis/animal/año) }\end{array}$ & Ventas (kg peso vivo) & $\begin{array}{l}\text { Producción/horas } \\
\text { Relación entre el valor } \\
\text { de la producción y } \\
\text { horas trabajadas }\end{array}$ \\
\hline $\begin{array}{l}\text { Capitalización de la } \\
\text { crianza (\$). Valor del } \\
\text { inventario de recursos } \\
\text { asociados a la crianza }\end{array}$ & $\begin{array}{c}\text { Suministro de forrajes, } \\
\text { (kg/días) }\end{array}$ & $\begin{array}{l}\text { Crías /reproductoras/ } \\
\text { año }\end{array}$ & $\begin{array}{c}\text { Ingresos/insumos } \\
\text { Relación entre los } \\
\text { ingresos y el valor de } \\
\text { los insumos }\end{array}$ \\
\hline \multirow[t]{2}{*}{$\begin{array}{l}\text { Área de instalaciones } \\
\qquad\left(\mathrm{m}^{2}\right)\end{array}$} & Insumos anuales (\$) & $\begin{array}{l}\text { Conteo de huevos de } \\
\text { parásitos (h.p.g). }\end{array}$ & $\begin{array}{l}\text { Ingresos/horas de } \\
\text { trabajo } \\
\text { Relación entre los } \\
\text { ingresos y las horas } \\
\text { trabajadas }\end{array}$ \\
\hline & $\begin{array}{l}\text { Horas de trabajo en la } \\
\text { crianza (horas/mes) }\end{array}$ & Ingresos/año (\$) & \\
\hline
\end{tabular}




\section{-Análisis estadístico}

Se empleó el (MEMI) ${ }^{(1)}$ para analizar el estado de cambio integral de los sistemas en los tres periodos de evaluación. Se seleccionó este modelo para reducir la dimensionalidad impuesta desde las 18 variables originales y simplificar el análisis de su evolución en el tiempo a partir de las combinaciones lineales de éstas, en forma de componentes.

La base de este modelo es el análisis multivariado de componentes principales con una variación en la conformación de la matriz de datos, que la hace reconocer el carácter longitudinal de la investigación (extendida en varios periodos). Para esto, se planteó una

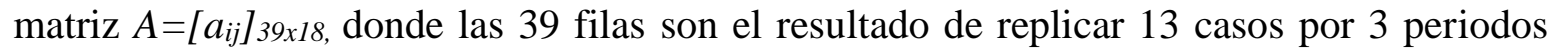
estudiados, y las 18 columnas se corresponden con las variables originales, declaradas en el Cuadro 2.

En el análisis de Componentes Principales, se extrajeron los componentes con autovalores superiores a 1 y se utilizó el método Varimax, para la rotación de los factores. El análisis de los resultados se basó en el comportamiento de los componentes extraídos y las variaciones de sus puntuaciones factoriales por casos y periodos de estudio. Según el marco de análisis del MEMI, estas puntuaciones factoriales, calculadas por el método de regresión, son consideradas como índices de impacto.

\section{Resultados y discusión}

El MEMI permitió reducir la dimensionalidad de las variables, incluidas en el seguimiento de los sistemas, a tres componentes (Cuadro 3).

La primer componente agrupó a variables relacionadas con la tenencia, comercialización y eficiencia económica, por tal motivo se etiquetó como "Tenencia y comercialización", la cual explicó el $58.4 \%$ de la varianza total. En este contexto, la tenencia es un indicador general que engloba a los rebaños tierras y recursos disponibles. El segundo componente explicó el $15.5 \%$ de la varianza total y asoció a variables de eficiencia productiva y control 
parasitario. Este componente se etiquetó como "Eficiencia productiva". El tercer componente se etiquetó como "Consumo y natalidad”, e integró las variables: consumo de productos caprinos y natalidad. Esta componente explicó el $8.0 \%$ de la variabilidad total. Con el análisis de estos tres componentes, se logró explicar el $82.0 \%$ de la variabilidad de los sistemas de crianza, durante todo el proceso.

Cuadro 3: Componentes principales extraídas del análisis de los impactos del proceso y su aporte a la varianza total

\begin{tabular}{lccc}
\hline & $\begin{array}{c}\text { Tenencia y } \\
\text { comercialización }\end{array}$ & $\begin{array}{c}\text { Eficiencia } \\
\text { productiva }\end{array}$ & Consumo y natalidad \\
\hline Año & 0.13 & -0.07 & 0.06 \\
No. de reproductoras & 0.96 & 0.14 & 0.07 \\
Sementales & 0.81 & 0.12 & 0.34 \\
Capitalización de la crianza (\$) & 0.98 & 0.01 & 0.11 \\
Área de instalaciones (m²) & 0.97 & 0.14 & 0.07 \\
Control parasitario (dosis) & 0.09 & 0.68 & 0.61 \\
Área de compensación (ha) & 0.88 & -0.18 & 0.15 \\
Suministro de forrajes (kg) & 0.96 & -0.09 & 0.04 \\
Insumos (\$) & 0.97 & -0.08 & 0.11 \\
Horas de trabajo en la crianza (horas & & & \\
mes) & 0.98 & -0.07 & 0.09 \\
Consumo (\$) & 0.41 & 0.40 & 0.70 \\
Ventas (\$) & 0.97 & 0.18 & 0.00 \\
Natalidad (\%) & -0.02 & 0.03 & 0.87 \\
Infestación parasitaria (HPG) & 0.02 & -0.80 & 0.29 \\
Ingresos (\$) & 0.96 & 0.08 & 0.00 \\
Producción/insumos & 0.43 & 0.72 & -0.08 \\
Producción/horas de trabajo & -0.42 & 0.73 & 0.00 \\
Ingresos/insumos & 0.88 & 0.24 & -0.12 \\
Ingresos/horas de trabajo & 0.70 & 0.33 & -0.18 \\
\hline Autovalores & 11.11 & 2.96 & 1.53 \\
Varianza explicada, \% & 58.45 & 15.58 & 8.04 \\
Varianza acumulada, \% & 58.45 & 74.03 & 82.08 \\
\hline
\end{tabular}

Respecto a la componente de mayor impacto -tenencia y comercialización- (Figura 2), se observó un patrón de evolución similar en ambas tipologías de sistemas. Los índices de impacto en esta componente, incrementaron su valor (puntuación factorial) desde la fase inicial (periodo de referencia) hasta el periodo de acompañamiento institucional. Sin embargo, con el cese del acompañamiento, se observó un proceso de colapso y renovación, en el cual, se redujeron los índices de impacto de esta componente, pero siempre en un estado superior respecto al inicio del proceso. La tipología sistemas de crianza familiar en patios y parcelas ${ }^{(4)}$, mostró niveles negativos en el índice de impacto de esta componente, y mayor tendencia a su reducción con el cese del acompañamiento. 
Figura 2: Evolución de los sistemas familiares de crianza de cabras criollas, según índices de impacto, en diferentes periodos del proceso de conservación de la cabra criolla cubana en la comunidad " 26 de Julio"
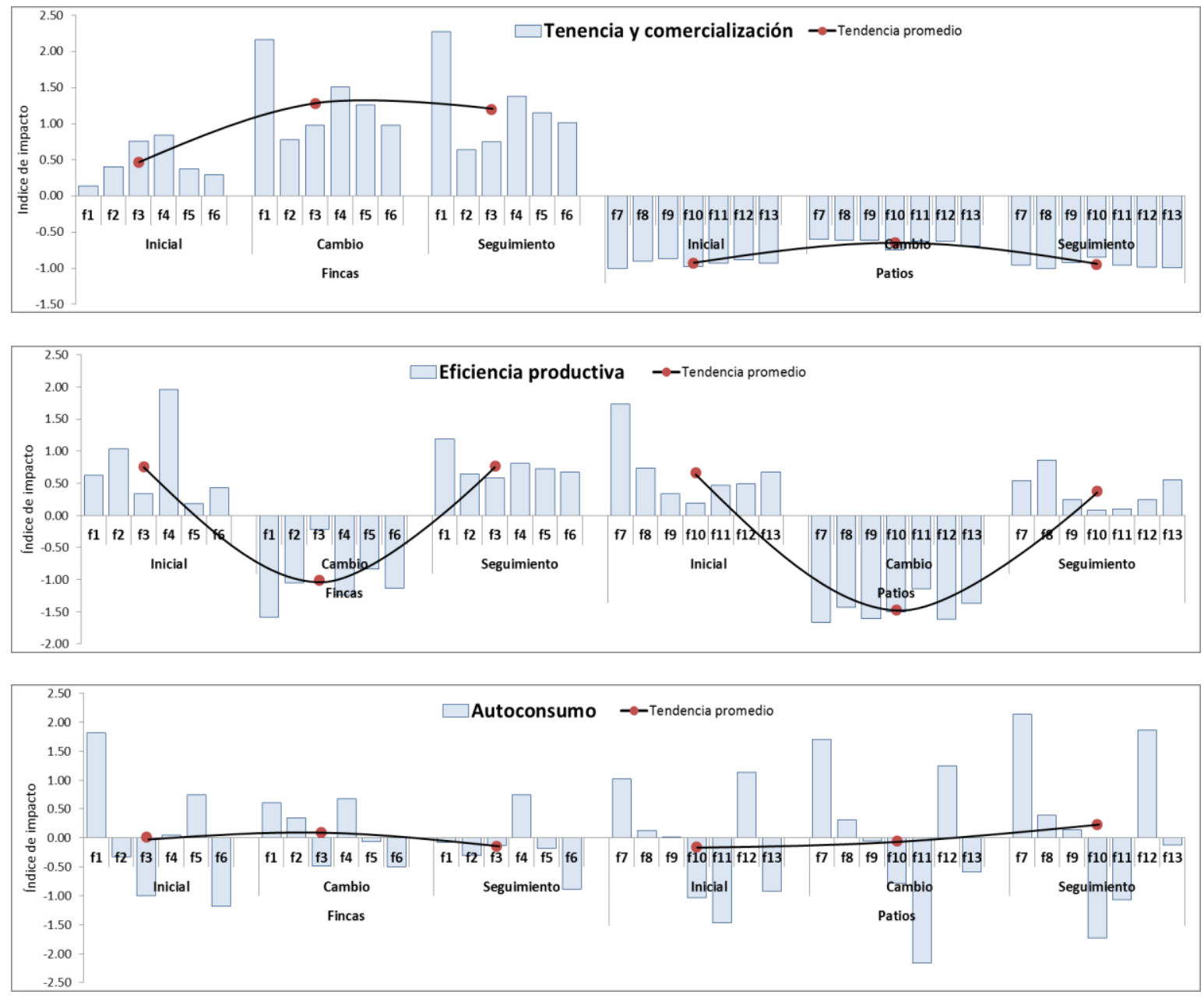

Este patrón de desarrollo, (crecimiento, colapso y renovación) se corresponde con el ciclo de renovación adaptativa de los sistemas complejos para una sola escala ${ }^{(5)}$, y el ciclo de "panarquía” para el análisis de estos procesos en múltiples ${ }^{(6)}$.

Con este marco de análisis, el periodo de referencia de los sistemas (2002-2003), se puede considerar en estado de organización. Dentro del ciclo adaptativo, los sistemas de crianza de cabras criollas, se encontraban en un pasaje de reorganización a explotación.

Este es el comienzo de los ciclos de panarquía. Según Matteucci ${ }^{(7)}$, en esta fase de la evolución de los sistemas complejos se producen los siguientes procesos: a) reducción del 
potencial de los sistemas, b) pérdida de algunos recursos acumulados, y c) ingreso de un legado de ciclos anteriores.

Estos tres procesos se pudieron constatar en los sistemas de crianza durante los años 2002 2003. En este periodo, el potencial de los sistemas de crianza estuvo bajo la influencia de limitantes internas, tales como el deficiente acceso a recursos y medios para la crianza, y la falta de percepciones en los criadores, sobre la problemática de la crianza y la extinción de este genotipo. También, se identificaron limitantes externas a los sistemas, relacionadas con la adversidad climática y la falta de políticas efectivas para impulsar la conservación y desarrollo de la raza criolla.

La pérdida de recursos acumulados se expresó en la reducción de la población de cabras criollas, la deforestación de la zona y la pérdida de la memoria bioculural. Se trata de la erosión del conocimiento tradicional asociado a la crianza y aprovechamiento sostenible de estas cabras.

Sin embargo, los saberes que aún persisten, la población caprina restante y las especies de pastos y arbustivas localmente adaptadas, representan el legado de largos procesos coevolutivos en ciclos anteriores. Estas son formas adaptativas a la variabilidad externa que representan a procesos acumulativos de aprendizaje de los sistemas mismos.

Durante el periodo de acompañamiento, se produjo una fase de explotación-crecimiento ${ }^{(7)}$. En este espacio, el potencial de cambio de los sistemas creció por el apoyo externo en recursos y capacitación. Los rebaños crecieron y se mejoró el acceso a recursos, para instalaciones y manejo (Cuadro 4). Además, el proceso de acompañamiento se enfocó al fortalecimiento de capacidades de criadores (desarrollo de nuevos conocimientos, habilidades y actitudes).

Cuadro 4: Comportamiento de las variables durante el proceso de seguimiento, acompañamiento y seguimiento, por tipologías de sistemas

\begin{tabular}{|c|c|c|c|c|c|c|c|}
\hline \multirow{2}{*}{ Indicador } & \multirow{2}{*}{$\begin{array}{l}\text { Estadí- } \\
\text { grafo }\end{array}$} & \multicolumn{3}{|c|}{$\begin{array}{c}\text { Crianza en } \\
\text { fincas }\end{array}$} & \multicolumn{3}{|c|}{$\begin{array}{c}\text { Crianza en } \\
\text { patios y parcelas }\end{array}$} \\
\hline & & inicial & Cambio & $\begin{array}{l}\text { Segui- } \\
\text { miento }\end{array}$ & inicial & Cambio & $\begin{array}{l}\text { Segui- } \\
\text { miento }\end{array}$ \\
\hline \multirow{2}{*}{$\begin{array}{l}\text { No. de } \\
\text { reproductoras }\end{array}$} & Media & 35 & 47 & 62 & 9 & 13 & 11 \\
\hline & $\mathrm{DE}$ & 3 & 8 & 14 & 2 & 2 & 3 \\
\hline \multirow{2}{*}{ Sementales } & Media & 2 & 2 & 2 & 1 & 1 & 1 \\
\hline & $\mathrm{DE}$ & 0.78 & 1.11 & 1.15 & 0.51 & 0.52 & 0.51 \\
\hline \multirow{2}{*}{$\begin{array}{l}\text { Capitalización de } \\
\text { la crianza, \$ }\end{array}$} & Media & 711.79 & 938.72 & 828.03 & 148.99 & 257.30 & 184.54 \\
\hline & $\mathrm{DE}$ & 102.64 & 144.59 & 147.10 & 33.08 & 50.68 & 53.87 \\
\hline \multirow{2}{*}{$\begin{array}{l}\text { Área de } \\
\text { instalaciones, } \mathrm{m}^{2}\end{array}$} & Media & 95 & 105 & 110 & 25 & 29 & 26 \\
\hline & $\mathrm{DE}$ & 8 & 11 & 17 & 5 & 6 & 5 \\
\hline
\end{tabular}




\begin{tabular}{|c|c|c|c|c|c|c|c|}
\hline \multirow{2}{*}{$\begin{array}{l}\text { Control parasitario } \\
\text { dosis }\end{array}$} & Media & 3.02 & 2.58 & 3.00 & 3.11 & 1.80 & 2.90 \\
\hline & $\mathrm{DE}$ & 0.87 & 0.12 & 0.37 & 0.87 & 0.33 & 0.78 \\
\hline \multirow{2}{*}{$\begin{array}{l}\text { Área de } \\
\text { compensación, ha }\end{array}$} & Media & 0.52 & 2.07 & 1.58 & 0.10 & 0.25 & 0.10 \\
\hline & $\mathrm{DE}$ & 0.07 & 0.84 & 0.64 & 0.13 & 0.05 & 0.13 \\
\hline \multirow{2}{*}{$\begin{array}{l}\text { Suministro de } \\
\text { forrajes, } \mathrm{kg}\end{array}$} & Media & 16.29 & 41.38 & 36.26 & 3.09 & 6.46 & 3.12 \\
\hline & $\mathrm{DE}$ & 3.53 & 9.18 & 13.03 & 0.88 & 1.16 & 0.86 \\
\hline \multirow{2}{*}{ Insumos, \$ } & Media & 232.56 & 319.84 & 300.87 & 71.42 & 121.71 & 87.96 \\
\hline & $\mathrm{DE}$ & 41.42 & 74.27 & 57.41 & 13.30 & 25.48 & 23.57 \\
\hline \multirow{2}{*}{$\begin{array}{l}\text { Horas de trabajo en } \\
\text { la crianza (horas } \\
\text { mes) }\end{array}$} & Media & 31.23 & 50.11 & 40.35 & 5.87 & 11.92 & 7.98 \\
\hline & $\mathrm{DE}$ & 7.16 & 9.62 & 6.13 & 0.91 & 3.04 & 1.32 \\
\hline \multirow{2}{*}{ Consumo, \$ } & Media & 90.77 & 72.53 & 89.82 & 69.84 & 66.10 & 70.38 \\
\hline & $\mathrm{DE}$ & 17.93 & 5.23 & 9.45 & 13.40 & 10.76 & 17.85 \\
\hline \multirow{2}{*}{ Ventas, \$ } & Media & 433.54 & 533.99 & 651.28 & 70.46 & 87.66 & 93.62 \\
\hline & $\mathrm{DE}$ & 136.52 & 97.32 & 145.74 & 16.91 & 24.68 & 18.91 \\
\hline \multirow{2}{*}{ Natalidad } & Media & 1.14 & 1.21 & 1.15 & 1.17 & 1.15 & 1.24 \\
\hline & $\mathrm{DE}$ & 0.12 & 0.06 & 0.10 & 0.17 & 0.35 & 0.26 \\
\hline \multirow{2}{*}{$\begin{array}{l}\text { Infestación } \\
\text { parasitaria HPG }\end{array}$} & Media & 783 & 300 & 650 & 543 & 193 & 650 \\
\hline & $\mathrm{DE}$ & 248 & 95 & 151 & 109 & 47 & 145 \\
\hline \multirow{2}{*}{ Ingresos, \$ } & Media & $2,199.36$ & $4,117.75$ & $4,906.35$ & 313.53 & 569.78 & 582.95 \\
\hline & $\mathrm{DE}$ & 733.68 & $1,287.89$ & $1,601.13$ & 52.48 & 102.75 & 92.74 \\
\hline \multirow{2}{*}{$\begin{array}{l}\text { Producción / } \\
\text { insumos }\end{array}$} & Media & 2.31 & 1.92 & 2.46 & 1.97 & 1.29 & 1.90 \\
\hline & $\mathrm{DE}$ & 0.74 & 0.14 & 0.13 & 0.19 & 0.24 & 0.21 \\
\hline \multirow{2}{*}{$\begin{array}{l}\text { Producción / horas } \\
\text { de trabajo }\end{array}$} & Media & 1.40 & 1.02 & 1.52 & 1.99 & 1.10 & 1.72 \\
\hline & $\mathrm{DE}$ & 0.20 & 0.13 & 0.11 & 0.29 & 0.13 & 0.26 \\
\hline \multirow{2}{*}{ Ingresos/insumos } & Media & 9.68 & 12.73 & 16.02 & 4.43 & 4.74 & 6.98 \\
\hline & $\mathrm{DE}$ & 3.87 & 1.57 & 2.12 & 0.47 & 0.51 & 1.82 \\
\hline \multirow{2}{*}{$\begin{array}{l}\text { Ingresos/horas de } \\
\text { trabajo }\end{array}$} & Media & 5.82 & 6.79 & 9.95 & 4.45 & 4.20 & 6.11 \\
\hline & $\mathrm{DE}$ & 1.39 & 1.26 & 1.74 & 0.25 & 1.23 & 0.49 \\
\hline
\end{tabular}

El acompañamiento al proceso, por parte de instituciones y organizaciones externas a la comunidad, resultó una condición necesaria, pero no suficiente, para el crecimiento de los sistemas. Este acompañamiento contribuyó a un querer crecer, saber crecer y poder crecer. Sin embargo, resultó necesario el legado acumulado por los sistemas (conocimiento tradicional, rebaños caprinos y vegetación), y las formas adaptativas establecidas (rusticidad de las cabras, pastos naturales persistentes, estrategias adaptativas de subsistencia, etc.). Se definió entonces que el crecimiento de estos sistemas, respondió a procesos constructivistas ${ }^{(8)}$, y no de simple depósito y suma de conocimientos, objetos y hechos.

El querer crecer, se asoció a la interacción entre las expectativas y estrategias de supervivencia, con las percepciones desarrolladas en los criadores sobre el problema de la extinción de la cabra criolla (percepción y sistematización del problema). En este sentido, 
se menciona que cada actor tiene su propia perspectiva del desafío de desarrollo, condicionada fuertemente por su sistema de intereses ${ }^{(9)}$, y la integración de los actores a los procesos de innovación agropecuaria se facilita en la medida que sus roles sociales se ven representados en dichos procesos ${ }^{(10)}$.

El saber crecer, se asoció a la interacción de las capacidades iniciales (fundamentalmente conocimiento tradicional) con las nuevas capacidades. Al analizarlo en los sistemas de crianza, es imprescindible considerar la efectividad de las diferentes herramientas de investigación-acción-aprendizaje participativo, las cuales, condujeron al desarrollo estructural y funcional de los sistemas.

Los principales cambios realizados en los sistemas de crianza, a partir de estas herramientas de investigación-acción y aprendizaje se enfocaron al crecimiento de áreas forrajeras con especies seleccionadas de forma participativa, y la introducción de prácticas de control y manejo de la reproducción, a partir de la experimentación participativa con campañas reproductivas. Se ha mencionado la marcada estacionalidad en el rendimiento de los pastos en esta región ${ }^{(11)}$, y argumentado la importancia de la gestión de reservas corporales, en sistemas de pequeños rumiantes, mediante el establecimiento de áreas de compensación ${ }^{(12)}$. En estas especies, según el ciclo productivo-reproductivo de las hembras, se producen variaciones significativas en el consumo de materia seca y demandas nutricionales; sin embargo, los momentos de mayor potencialidad de consumo y requerimientos de materia seca, coinciden con la menor disponibilidad de pastos. También se ha señalado ${ }^{(13)}$ la influencia positiva del control y manejo reproductivo, sobre el desempeño general de los sistemas de producción caprina. Existe coincidencia ${ }^{(12,13)}$ en que la combinación efectiva del manejo de la reproducción con la estrategia de gestión de reservas corporales son determinantes en el desempeño de los sistemas de crianza de ganado menor.

El resultado más relevante para el crecimiento de los sistemas, fue el registro oficial de ejemplares criollos. Con este registro, se creó un valor agregado, que incrementó el precio de venta de los animales en desarrollo de $\$ 8.00$ a $\$ 12.00$ pesos cubanos el kilo, lo que incidió en el esquema de comercialización y la respuesta económica (relación ingresos/insumos e ingresos/horas de trabajo.

La complementación logística y financiera de actores acompañantes, (Programa de Pequeñas Donaciones del Fondo para el Medio Ambiente Mundial), potenció el poder crecer. Este aporte de complementación, se reflejó en los cambios estructurales de los sistemas, tales como el incremento de la capitalización e instalaciones, además posibilitó el desarrollo del proceso de formación de capacidades para el saber crecer. Estos aspectos, permitieron asumir que los cambios de los sistemas de crianza resultaron procesos acumulativos de aprendizaje ${ }^{(14)}$. 
Desde el marco de análisis sistémico ${ }^{(15)}$, se observó la coevolución entre los componentes de la biodiversidad y los componentes culturales-tecnológicos. Al valorar los cambios en elementos estructurales de los sistemas, se determinaron diferencias evolutivas, entre tipologías de sistemas. Al mismo tiempo, las tipologías, condicionaron las probabilidades de sus componentes, según su capacidad de aprovechar el contexto habilitador para el desarrollo.

En toda esta fase de crecimiento, el comportamiento de los sistemas se hizo más dependiente de las interacciones entre los componentes desarrollados (rebaño, manejo, capitalización, áreas forrajeras, insumos, comercialización). Este proceso se denomina desarrollo de la conectividad, y tal conectividad propicia una mayor vulnerabilidad de los sistemas ante las fluctuaciones del entorno y menor capacidad de resiliencia ${ }^{(7)}$. Esto colocó a ambas tipologías de sistemas de crianza en un punto crítico o límite crítico para la agregación, donde su crecimiento sobrepasó un umbral de estabilidad. Este límite para la agregación, es teórico y explica el colapso de los sistemas. Se ha fundamentado ${ }^{(5)}$ que este límite se asocia a algún cambio externo a los sistemas, que en este caso sería la suspensión del acompañamiento.

El proceso teórico de renovación, posterior al colapso, se fundamentó a partir del análisis combinado de los índices de impacto de las dos primeras componentes, que explicaron el $74.0 \%$ de la varianza total (Figura 1).

El primer indicador de renovación, fue el comportamiento del componente I, Tenencia y comercialización, el cual quedó en un estado superior después del cese del acompañamiento institucional respecto al estado inicial, especialmente en la tipología de crianza en fincas. Las reducciones en los índices de impacto de este componente, se produjeron fundamentalmente por las variables estructurales (instalaciones capitalización, áreas forrajeras). Sin embargo, las variables de comercialización y relación ingresos/insumos e ingresos/horas de trabajo, mostraron menor tendencia a la reducción.

El segundo indicador de renovación se observó en el análisis de la componente II (eficiencia productiva). El crecimiento de los sistemas, afectó su eficiencia productiva (relación producción/insumos producción/horas de trabajo). Al retirarse el apoyo externo, se produjo una pérdida del crecimiento que dependía del mismo, y quedaron los cambios compatibles con las racionalidades socioeconómicas del sistema. Esto, provocó una recuperación de la eficiencia productiva en ambas tipologías.

El mantener las racionalidades socioeconómicas en el manejo de la biodiversidad desde la perspectiva local no es resistencia al cambio, es ser consecuente con la seguridad alimentaria y la capacidad de adaptación a perturbaciones externas, tanto económicas como ecológicas. Esta recuperación, es además un indicador de que los sistemas evolucionaron a un estado superior, avalando un proceso de reorganización y no de involución ${ }^{(16)}$. 
Al analizar los procesos de reorganización de los sistemas complejos, se propuso una explicación a partir del concepto de "criticidad auto organizada"(6), en tanto que Holling ${ }^{(17)}$ menciona que "la conectividad de un sistema altamente integrado se incrementa hasta un punto de sobreconexión o hiperconectividad, donde el propio sistema se convierte en una estructura rígida autoconstruida... En esa situación, el sistema se vuelve muy vulnerable debido a su incapacidad de respuesta, lo que llevará indefectiblemente a un colapso seguido de una reorganización... los recursos residuales se reorganizan dejando oportunidades potenciales para la experimentación y la innovación".

La idea de que los sistemas han alcanzado un límite crítico implica que la conectividad y la vulnerabilidad comenzarán a disminuir gradualmente, mientras que su capacidad de resiliencia comenzará a desarrollarse. No se trata de un proceso de retroceso o involución, sino de un proceso de liberación del potencial acumulado. Por el contrario, se crea el espacio para la incorporación de nuevos modelos y el cambio de paradigma, o sea, para la innovación ${ }^{(5,7)}$. En esta nueva fase, los sistemas manifestaron capacidad para regenerarse, tratando de conservar sus lógicas (aportes a las estrategias de subsistencia y racionalidades socio-económicas), en una especie de conservación creativa, denominada resiliencia socioecológica ${ }^{(18)}$.

En este caso se verificó el desarrollo armónico de dos conceptos considerados antagónicos por el pensamiento simplista: conservación y revolución. Estos conceptos deben de ser asociados en los problemas complejos bajo el principio de que para conservar hay que cambiar $^{(19)}$. La resiliencia de un socio-ecosistema debe ser entendida como su capacidad para hacer frente a los cambios, no para resistirse a ellos - pues son inevitables-. Se trata de la habilidad de un socioecosistema para absorber creativamente la perturbación y reorganizarse, al tiempo que experimenta el cambio, reteniendo esencialmente las mismas funciones, estructura, identidad y retroalimentaciones ${ }^{(20)}$.

El comportamiento de los índices de impacto en el componente III (Consumo y natalidad) no mostró una tendencia definida durante el proceso de conservación. La variabilidad asociada a este componente resultó muy diversa, en ambas tipologías, lo que demuestra heterogeneidad en las estrategias y medios de subsistencia familiares. Esta heterogeneidad, es clave para comprender que los sistemas campesinos, también están sujetos a la incertidumbre y lo imprevisto, lo cual no resiste un pensamiento simplista y reduccionista $^{(20)}$.

La comprensión del concepto de resiliencia socio-ecológica, aporta una nueva perspectiva de análisis, desde modelos simples de relación causa-efecto, hacia sistemas complejos ${ }^{(21)}$. Esto da fundamento a promover la mejora de estos sistemas de crianza, respetando, comprendiendo y fomentando sus lógicas de adaptación, que son verdaderos procesos creativos de autosubsistencia en contextos complejos. 


\section{"Conclusiones e implicaciones}

La evolución de los sistemas de crianza de cabras criollas se caracterizó por el crecimiento de las variables asociadas a la tenencia y comercialización, con una reducción de su eficiencia productiva durante el periodo de acompañamiento institucional. Con el cese de dicho acompañamiento, los sistemas se reorganizaron y asimilaron creativamente los cambios tecnológicos consistentes con sus respectivas racionalidades socioeconómicas y la recuperación de las variables asociadas a su eficiencia. Las tipologías de sistemas siguieron patrones diferentes en estos procesos. Los sistemas de crianza en fincas, manifestaron mayor capacidad para el crecimiento, mientras, los sistemas de crianza en patios y parcelas tuvieron mayor tendencia a conservar su racionalidad socioeconómica de supervivencia.

\section{N Agradecimientos}

Al Programa de Pequeñas Donaciones (PPD) del Fondo para el Medio Ambiente Mundial, en especial su oficina en Cuba, por el financiamiento, acompañamiento y apoyo a la iniciativa de conservación de la cabra Criolla Cubana que hizo posible esta investigación. Al Programa de Innovación Agropecuaria Local (PIAL) por su apoyo logístico, financiero y metodológico a esta experiencia.

\section{- Literatura citada}

1. Torres V, Ramos N, Lizazo D, Monteagudo F, Noda A. Modelo estadístico para la medición del impacto de la innovación o transferencia tecnológica en la rama agropecuaria. Rev Cub Cienc Agríc 2008;42(2):133-139. 
2. Capote J, Fresno M. La colonización americana y el ganado caprino. Biodiversidad Caprina Iberoamericana. 2016.

3. Comisión Nacional de Recursos Genéticos de la República de Cuba. Informe de País sobre la Situación Nacional de los Recursos Zoogenéticos en Animales de Granja. La Habana, Cuba: FAO; 2003.

4. La O M. Estudio de conservación de la cabra criolla cubana en la sub-cuenca Cautillo del Valle del Cauto [tesis doctorado]. Instituto de Ciencia Animal. Mayabeque, Cuba; 2013.

5. Calvente AM. Ciclo de renovación adaptativa. Universidad Abierta Interamericana, Centro de Altos Estudios Globales. http://www. sustentabilidad. uai. edu. ar/pdf/cs/UAIS-CS-200-004; 2007. Consultado 28 Sep, 2016.

6. Allen CR, Angeler DG, Garmestani AS, Gunderson LH, Holling CS. Panarchy: theory and application. Ecosystems 2014;17(4):578-589.

7. Matteucci SD. Panarquía y manejo sustentable. Fronteras (Rev del GEPAMA) 2004;(3):1-12.

8. Guevara-Hernández F, Hernández A, Pinto R, Gómez H, Medina F, Martínez B. La innovación como proceso social: de la teoría y los conceptos a las prácticas para el desarrollo rural. En: Rodríguez LL, Guevara HF, editores. Innovación y desarrollo rural: Reflexiones y experiencias desde el contexto cubano. 2da ed. IIA "Jorge Dimitrov". UNACH. RED A.C. ACSUR Las Segovias. Bayamo, Granma. Cuba; 2011:1-10.

9. Rubiano MFG, Medrano JCM, Medel RR, Carvajal REG. Los sistemas de innovación y su impacto en el desarrollo territorial. Rev Mex Cienc Agr 2016: 3143-3152.

10. Fonseca M, Guevara-Hernández F. Del modelo convencional a la innovación como proceso social: el papel de la ciencia y los actores del desarrollo. En: Rodríguez LL, Guevara HF, editores. Innovación y desarrollo rural: Reflexiones y experiencias desde el contexto cubano. 2da ed. IIA "Jorge Dimitrov". UNACH. RED AC. ACSUR Las Segovias. Bayamo, Granma. Cuba 2011:16-28.

11. Vega AM, Herrera RS, Torres V, Lamela L, Montejo I, Santana A, Cino DM. Performance of replacement Cuban Charolais females in a silvopastoral system compared to a monoculture. Cuban J Agr Sci 2016;50(1):51-59.

12. Fuentes, NF. Contribución a la producción sostenible en rebaños ovinos en el Valle del Cauto. Ann Acad Cienc Cuba. 2014. 
13. Costa P. Mejora de indicadores reproductivos en cabras Saanen y Alpinas en condiciones de montaña mediante adecuaciones en los procedimientos tecnológicos [tesis maestría]. Cuba: Universidad de Granma; 2001.

14. Machado H, Suset A, Martín GJ, Funes-Monzote FR. Del enfoque reduccionista al enfoque de sistema en la agricultura cubana: un necesario cambio de visión. Pastos y Forrajes 2009;32(3):1-20.

15. Vara-Sánchez I. Cuéllar-Padilla M. Biodiversidad cultivada: una cuestión de coevolución y transdisciplinariedad. Ecosist 2013;22(1):1-5.

16. Ecosistemas del Milenio (EM). Evaluación de ecosistemas del milenio. Ecosistemas y bienestar humano: Síntesis de biodiversidad. Millennium Ecosystem Assessment. 2005.

17. Holling CS. Understanding the complexity of economic, ecological and social systems. Ecosystems 2002;4:390-405.

18. Escalera-Reyes J, Ruiz-Ballesteros E. Resiliencia socioecológica: aportaciones y retos desde la antropología. Rev Antrop Soc 2011;20:109-135.

19. Casanova-Pérez, L, Martínez-Dávila JP, López-Ortiz S, Landeros-Sánchez C, LópezRomero G, Peña-Olvera B. Enfoques del pensamiento complejo en el agroecosistema. Interc 2015;40;(3):210.

20. Walker B, Salt D. Resilience thinking: sustaining ecosystems and people in a changing world. USA: Island Press; 2012.

21. Davidson-Hunt I, Idrobo C, Pengelly R, Sylvester O. Anishinaabe adaptation to environmental change in northwestern Ontario: a case study in knowledge coproduction for nontimber forest products. Ecol Soc 2013;18(4)44-54. 